\title{
Editorial: Evolution of Postembryonic Development
}

\author{
Nico Posnien ${ }^{1 *}$, Patrícia Beldade ${ }^{2}$ and Fernando Casares ${ }^{3}$ \\ ${ }^{1}$ Department of Developmental Biology, Göttingen Center for Molecular Biosciences (GZMB), \\ Johann-Friedrich-Blumenbach-Institute of Zoology and Anthropology, University Göttingen, Göttingen, Germany, ${ }^{2}$ Faculty of \\ Sciences, Centre for Ecology, Evolution, and Environmental Changes, University of Lisbon, Lisbon, Portugal, ${ }^{3}$ Andalusian \\ Center for Development Biology (CABD), Spanish National Research Council (CSIC), Universidad Pablo de Olavide, Seville, \\ Spain
}

Keywords: evolution and development, postembryonic development, comparative genomics and transcriptomics, geometric morphometrics, genetics, metamorphosis, hormonal control

Editorial on the Research Topic

Evolution of Postembryonic Development

\section{INTRODUCTION}

Adult traits, such as morphology, physiology and behavior are defined during embryonic and postembryonic development, and the diversification of such traits is a result of changes in developmental programs. In organisms with direct development, selection on adult traits will result in changes in genes and processes involved in embryonic development. In contrast, in organisms that undergo metamorphosis (e.g., holometabolous insects) or grow throughout their entire life (e.g., crustaceans), variation in adult traits can result from changes in different life stages. Investigating the proximal and ultimate mechanisms shaping postembryonic development is an exciting endeavor that has been boosted by a series of technological advances. For example,

\section{OPEN ACCESS}

Edited and reviewed by: Mark A. Elgar

The University of Melbourne, Australia

*Correspondence: Nico Posnien nposnie@gwdg.de

Specialty section:

This article was submitted to

Evolutionary Developmental Biology,

a section of the journal

Frontiers in Ecology and Evolution

Received: 24 January 2022 Accepted: 03 February 2022 Published: 24 February 2022

Citation:

Posnien N, Beldade P and Casares F (2022) Editorial: Evolution of Postembryonic Development. Front. Ecol. Evol. 10:859670. doi: 10.3389/fevo.2022.859670 recent advances in sequencing (e.g., transcriptomics, genomics) and genetic engineering (e.g., CRISPR/Cas9, RNAi) technologies allow going beyond well-established model organisms to enhance our understanding of how biological diversity is generated. Major innovations in imaging technologies and mathematical frameworks (e.g., Geometric Morphometrics) to describe complex morphological features further enable researchers to study postembryonic developmental processes on a mechanistic and quantitative level.

This Research Topic on "Evolution of Postembryonic Development" contributes primary data articles, perspectives and reviews, which collectively highlight the technical and conceptional challenges and opportunities in studying developmental process beyond embryonic stages. All contributions focus on the impact of variation in postembryonic development on variation in adult traits at evolutionary and individual scales (i.e., plasticity).

\section{MANY DEVELOPMENTAL PROCESSES CAN ONLY BE STUDIED IN POSTEMBRYONIC STAGES}

Many developmental processes are restricted to postembryonic stages and thus require the analysis of later stages of an organism's life history. For instance, Beer and Helfrich-Förster present novel insights into the development of the circadian clock. They document a temporal shift in the formation of the cell types responsible for circadian behaviors which are already present and fully functional in newly eclosed non-social (i.e., solitary) bees, but only develop during adult stages in a social bee. Similarly, parts of the nervous system and muscles develop during 
larval stages in ribbon worms (Nemertea), a small phylum of worm-shaped, mostly marine Lophotrochozoans. von Döhren presents comparative data on larval nervous system and muscle development in these worms. While the postembryonic development of the FMRFamide-like nervous system seems to be conserved among the studied species, the author observed highly variable development of the body wall musculature. Also, the beautiful color patterns and eye spots of butterfly and moth wings are the result of postembryonic developmental processes. Tendolkar et al. generated CRISPR/Cas9 mediated somatic Ultrabithorax knock-out clones in three species to study the impact of loss of function of this Hox gene on adult fore- and hindwing morphology. The data confirmed a role of Ultrabithorax in defining hindwing identity and effects on various aspects of wing morphology, such as scale derivatives, color patterns, eyespots, and wing structure. The authors conclude that Ultrabithorax has pleiotropic functions during postembryonic wing development, compatible with a micromanaging role of this Hox gene.

\section{PLASTICITY OF POSTEMBRYONIC DEVELOPMENT}

Developmental processes are affected by environmental cues resulting in plastic responses. Reis et al. applied geometric morphometrics to study plasticity in wing shape, as well as size and shape relationships in three Diptera species. The size and shape of fly wings is defined during postembryonic development in wing imaginal discs. Controlled variation in rearing temperature and larval density resulted in conserved and species-specific changes in wing size and shape, which is discussed in the context of different mating behaviors of the three fly species. May et al. demonstrate in Drosophila melanogaster that the larval diet has a major impact on adult gene expression. Intriguingly, different larval food compositions affected males and females differently. While genes affected by larval diet are related to reproduction processes in females, gene expression in males is related to nutrient sensing and metabolic processes. The authors raise the important question about whether and how these gene expression differences relate to the fitness of young adults.

\section{METAMORPHOSIS AND SEXUAL MATURATION ARE POSTEMBRYONIC PROCESSES IN INSECTS}

Metamorphosis and sexual maturation are key postembryonic processes in insects. Gegner et al. revealed extensive modulation of gene expression during metamorphosis in the tobacco hornworm Manduca sexta. They show that about half of the genes in the genome are differentially expressed between late larval and adult stages. Intriguingly, part of this transcriptomic remodeling is associated with epigenetic methylation marks that may have an impact on chromatin architecture through histone modification mechanisms. Various hormones and receptors regulate postembryonic molts and the onset of metamorphosis. Korb et al. studied the effect of juvenile hormone induction on postembryonic molts in termites and linked morphological variants to effects on juvenile hormone related gene expression. Their data confirms a previously suggested model proposing that juvenile hormone levels between postembryonic molts contribute to morphological diversity in termites. Taubenheim et al. provide a comprehensive review of the function and evolution of nuclear receptors, such as steroid receptors and thyroid receptors in vertebrates and ecdysone receptors in insects, which coordinate key postembryonic developmental transitions. The authors argue that future research should focus on how nuclear receptors integrate intrinsic and extrinsic environmental cues to control developmental transitions and they suggest that a broad taxonomic sampling with a special emphasis on early branching bilaterians and cnidarians is a promising route.

\section{CHALLENGES OF CURRENT VIEWS AND FUTURE DIRECTIONS}

In their Mini Review, Fusco and Minelli summarize findings from the fossil record, comparative morphology of extant taxa and gene expression studies in the context of segment addition after embryonic stages are completed (i.e., anamorphosis). Based on these data, they conclude that in many cases a clear distinction between embryonic and postembryonic stages is not as straightforward as previously thought. To understand the evolution of skeletal development and to reveal developmental modularity, ossification sequences are extensively studied in vertebrates. Rose discusses potential caveats of using these methods in amphibians and he calls for more integrative approaches that also include physiology and behavioral insights. White et al. summarize the current knowledge about sutures, fibrous joints that separate the cranial bones in mammals. These sutures play important roles during skull development, and they are also important for the function of skull bones, such as joint mobility during feeding. The authors argue that a thorough understanding of the evolution of adult skull morphology in mammals must integrate data on the development and function of sutures.

\section{CONCLUSIONS}

The articles collected in this Research Topic highlight the importance of postembryonic developmental processes for a comprehensive understanding of the evolution of development and the mechanisms underlying phenotypic diversity present in nature. The breadth of methods applied range from thorough morphological descriptions to the integration of next generation sequencing data and therefore provide an excellent roadmap for future studies. It will be exciting to expand the interdisciplinary experimental toolkit to apply it to as many organisms as possible to reveal common and diverse aspects of postembryonic development. 


\section{AUTHOR CONTRIBUTIONS}

All authors co-edited the Research Topic and contributed to the article. The submitted version of this editorial is approved by all authors.

\section{ACKNOWLEDGMENTS}

We want to thank all authors who contributed their exciting data, thoughts, and ideas to this Research Topic. Thanks go to the Frontiers Editorial team that has been helpful throughout the process. Finally, special thanks to the external peer reviewers who invested considerable time to provide constructive feedback on the manuscripts in this issue.
Conflict of Interest: The authors declare that the research was conducted in the absence of any commercial or financial relationships that could be construed as a potential conflict of interest.

Publisher's Note: All claims expressed in this article are solely those of the authors and do not necessarily represent those of their affiliated organizations, or those of the publisher, the editors and the reviewers. Any product that may be evaluated in this article, or claim that may be made by its manufacturer, is not guaranteed or endorsed by the publisher.

Copyright (c) 2022 Posnien, Beldade and Casares. This is an open-access article distributed under the terms of the Creative Commons Attribution License (CC BY). The use, distribution or reproduction in other forums is permitted, provided the original author(s) and the copyright owner(s) are credited and that the original publication in this journal is cited, in accordance with accepted academic practice. No use, distribution or reproduction is permitted which does not comply with these terms. 